\title{
Semi-blind Unmixing of Hyperspectral Image by Eigenvalue based Virtual Dimensionality Estimation
}

\author{
Samiran Das
}

\begin{abstract}
Hyperspectral images provide an abundance of rich spectral information and thus facilitate accurate classification, object identification. The band selection process circumvents the computational inconvenience in dealing with too many spectral bands by identifying some specific informative bands before further processing. In this paper, we propose a deep subspace clustering framework followed by a multi-criteria based selection method. The proposed deep subspace clustering approach uses the ability of the deep neural network to identify the inherent manifold structure present in the data with high accuracy. We incorporate pq-norm based sparsity in the subspace clustering framework. Compared to the traditional subspace clustering methods, deep subspace clustering methods can better characterize the complex non-linearities present in the real hyperspectral images and performs clustering accordingly. In the succeeding stage, we select the representative bands from each cluster using structural information and information content. The use of multiple criteria helps in selecting more informative and discriminative bands. We conduct real image experiments and observed that our proposed band selection approach is more informative compared to the other approaches. We also evaluate the efficacy of the proposed band selection method using classification and unmixing performance. method using classification and unmixing performance.
\end{abstract}

Index Terms-Hyperspectral unmixing, Semi-blind unmixing, Eigenvalue analysis, Virtual dimensionality

\section{INTRODUCTION}

Hyperspectral imaging is an advanced technology which has transformed remote sensing domain significantly. It captures detailed high-resolution spectral information at hundreds of bands in the electromagnetic region (1)-(2) spreading from visible to far infra-red. The wealth of spectral information leads to superior discriminative capability. It has specific application in agricultural, geological and environmental studies, which require accurate object identification and land cover mapping. The mathematical process central to object identification is called unmixing (3)-(? ).

Researchers have tried to solve unmixing task employingconvex geometry, sparse inversion, convex optimization, Bayesian learning etc. Every unmixing method requires precise estimation of the number of endmembers (4) present in the image or an estimation of the virtual dimensionality. Although virtual dimensionality (VD) is central to the unmixing framework, many unmixing works overlooked the step. Other works considered VD estimation as the initial stage. This paper proposes a virtual dimensionality estimation method, which central to our proposed dictionary pruning framework (5). To the best of our knowledge, this is the first unmixing method which uses VD estimation directly for library pruning.

S. Das is with School of Computer Science, University of Petroleum and Energy Studies
In our work, we compute the abundance matrix after library pruning by an optimization formulation which uses image structural property, sparsity and low-rankness of the abundance matrices as regularization terms. We also smoothen the norms to achieve better convergence property.

Our proposed dictionary pruning method offers few distinct benefits such as-

1) The proposed method demonstrates better accuracy in both VD estimation and dictionary pruning

2) The proposed framework is relatively simpler as it which does not require any tuning parameter apart from the data and spectral library, which is always desirable

3) Our proposed dictionary pruning approach has achieved improved performance in the presence of noise, compared to the existing methods

4) The simplicity of the proposed framework leads to significantly reduced computational runtime.

We organize the paper into the following pattern. Section 2 outlines the signal model for the unmixing task. Section 3 presents a brief overview of existing unmixing workflows, section 4 illustrates our proposed VD estimation and dictionary pruning method, section 5 presents the experimental results and section 6 includes the conclusion and future scope.

\section{Signal Model For UnMixing}

A hyperspectral image is represented as a linear mixture of the whole spectral library. The reflectance of the $i$-th pixel can be written as-

$$
x_{i}=m_{i} D+w_{i}
$$

where, $m_{i}$ is the abundance of $i$-th pixel and $w_{i}$ is the noise in pixel $i$ and $D$ is the spectral library. The hyperspectral image can be written as-

$$
X=M D+W
$$

where, $X=\left[x_{1}, x_{2}, \cdots x_{N}\right]$ is the hyperspectral image, which contains $N$ pixels $M=\left[m_{1}, m_{2} \cdots m_{N}\right]$ is the mixing matrix or abundance matrix

$D=\left[d_{1}, d_{2} \cdots d_{K}\right]$ and $W=\left[w_{1}, w_{2} \cdots w_{N}\right]$ is the noise matrix

Sparse unmixing algorithms obtain the abundance matrix $M$ which minimizes the reconstruction error, maximize sparsity while satisfying other regularization term.

$$
\begin{array}{r}
\arg \min _{D}\|X-M D\|_{2}+\lambda\|M\|_{q} \\
\text { where }, 0 \leq q \leq 1
\end{array}
$$

Here, the first term indicates reconstruction error, whereas the second term represents sparsity of abundance matrix. 
Dictionary pruning algorithms identify the specific spectral library atoms which also leads to a compact representation of the image. The $i$-th pixel is expressed as a linear combination of the pruned library $\hat{D}$.

$$
x_{i}=\hat{m}_{i} \hat{D}+\hat{w}_{i}
$$

where, the pruned library $\hat{D}=\left[\hat{d}_{1}, \hat{d}_{2}, \cdots \hat{d}_{r}\right]$ consists of $R$ elements $(R \ll K)$. Dictionary pruning algorithms with unity probability of detection and $(R=P)$ designate perfect result $\hat{m}_{i}$ represents the abundance of the pruned library endmembers in the $i$-th pixel and $w_{i}$ is the noise present in the $i$-th pixel. The complete image is expressed as-

$$
X=\hat{M} \hat{D}+\hat{W}
$$

here, $\hat{M}=\left[\hat{m}_{1}, \hat{m}_{2}, \cdots, \hat{m}_{r}\right]$ represents the abundance matrix $\hat{W}=\left[\hat{w}_{1}, \hat{w}_{2}, \cdots, \hat{w}_{N}\right]$ is the noise term.

In the context of hyperspectral unmixing, virtual dimensionality (VD) and the number of endmembers are analogous. The term refers to the number of spectrally distinct elements present(6). Existing estimation methods include- Harsanyi Ferrand Chang method (HFC) (6), hyperspectral subspace estimation (7), empirical automatic estimation (ELM) (8), eigenvalue thresholding (9), entropy estimation of eigenvalue (10), low rank subspace representation (11), Akaike Information Criteria (AIC) (12), minimum description length (MDL) (13), maximal orthogonal complement algorithm (MOCA) (14), high-order statistics (HOS)-HFC (15), tensor-based estimation (4), Stein's unbiased rick estimator (16), convex set-based estimation (17) etc. However, these algorithms possess some inherent limitations, which make them unsuitable for efficient library pruning. Some of these limitations are-

- Some of these algorithms require a high amount of computational runtime

- Most of the algorithms require proper selection tuning parameters or threshold as input

- Accuracy of these algorithms degrade in the presence of noise and high mutual coherence of spectral library

- These algorithms cannot give accurate estimation in the presence of rare endmembers

- They require a sufficient number of observations. Hence, it can not give an accurate estimation for image patches, where the number of pixels is comparatively less

An ideal virtual dimensionality estimation method should possess the following attributes so that we utilize it in dictionary pruning process-

1) Accurate estimation in the presence of rare endmembers: When we merge a specific spectral library element with the original data, the library elements is considered as a rare endmember, as it is present in very few observations. The attribute mentioned above gives rise to a straight forward way to identify whether the library element is an image endmember or not.

2) Accurate estimation for a lower number of observations: Most of the VD estimation methods require a large number of samples for accurate estimation. As a consequence, these methods cannot infer VD from small patches. An ideal VD estimation approach should also be able to provide accurate estimation from image patches too. This attribute ensures accurate estimation of virtual dimensionality in even image patches.

\section{SEMI-BLIND UNMIXING}

Present dictionary pruning methods for semi-blind unmixing include- greedy algorithms, convex optimization, sparse regression and signal subspace projection etc. Customary greedy algorithms include- orthogonal matching pursuit (OMP) (18), OMP Star (19), covariance similarity-based unmixing (20), subspace matching pursuit (SMP) (21), compressive sampling matching pursuit (CoSaMP) (22), sparsity measure-based approach (23), simultaneous orthogonal matching pursuit (SOMP) (24), sparse unmixing using spectral a priori information (SUnSPI) (25), centralized collaborative unmixing (26), simplex volume ratio appraoch (27), deblurring and sparse unmixing (28), regularized simultaneous forward-backward greedy algorithm (RSFoBa) (29) etc. Signal subspace learning approaches include- MUlti SIgnal Classification-Collaborative Sparse Regression algorithm (30) and Robust MUSIC(30). Other approaches such as multi-objective unmixing (? ), spectral library adjustment approach (? ) employ convex optimization for unmixing. MUSIC-CSR algorithm (30) use HySIME algorithm (7) for estimation of signal subspace. In the next stage, each spectral library element is projected on signal subspace and the projection error is recorded. RMUSIC algorithm (30) presents an improved noise robust version of the inversion process, which elucidates the mismatch between spectral library elements and actual image endmembers.

However, we observed the following deficiencies in the traditional semi-blind unmixing algorithms-

- Size of the pruned library is often much higher than the actual number of endmembers

- Some algorithms require a high amount of computational time

- Inversion process leads to sparse abundance images, most of which have much significance

- They often display unsatisfactory performance when the spectral library has high mutual coherence

Mutual coherence of a spectral library represents the maximum similarity between any pair of library elements, where the methods computed similarity in terms of the inner product. When a spectral library contains high mutual coherence, the semi-blind unmixing methods typically identify the endmembers with similar reflectance profile. This false identification reduces the sparsity of the obtained abundance matrix. Mutual coherence of a spectral library of size $K \times L$ is computed by-

$$
\mu(\mathbf{D})=\arg \max _{1 \leq i, j \leq K, i \neq j} \frac{\left|d_{i}{ }^{T} d_{j}\right|}{\left\|d_{i}\right\|_{2}\left\|d_{j}\right\|_{2}}
$$

An efficient dictionary pruning based unmixing algorithm should not require the number of endmembers or other tuning parameters as input and should obtain a pruned library with the optimum size. The cardinality of the pruned library should equal the actual number of endmembers. Ideally, the performance of the unmixing framework should be less affected 
by other parameters such as- the degree of mixing, mutual coherence, noise etc.

\section{Proposed Semi-Blind UnMIXING WORKFlow}

Our proposed semi-blind unmixing workflow constitute of three stages-

1) Noise removal by multi linear regression (31)

2) Dictionary pruning by GAP index based virtual dimensionality estimation

3) Sparse inversion using sparse unmixing by augmented Lagrangian (SUnSAL) (18)

\section{A. Noise Removal by Multi Linear Regression}

We have used multi linear regression framework (31) for noise removal, as it has been found to be very effective in the hyperspectral setting.

\section{B. Dictionary pruning by GAP index based virtual dimension- ality estimation}

The proposed library pruning method utilizes efficient VD estimation by eigenvalue based GAP index. The method computes the eigenvalues $\left(\lambda_{i}\right)$ of the data covariance matrix. The eigenvalues are arranged in descending order with a longtailed pattern, and he higher eigenvalues correspond to signal content, and the lower eigenvalues represent noise components or insignificant sources (32). However, instead of finding a hard threshold for separation of signal components from noisy components (9), we propose an alternate index, termed GAP index (33) which is defined by-

$$
I_{G A P}(K)=\frac{\sigma_{\hat{\lambda}_{K+1}: \hat{\lambda}_{L-1}}}{\sigma_{\hat{\lambda}_{K}: \hat{\lambda}_{L-1}}}
$$

where, $\tilde{\lambda}_{i}=\lambda_{i}-\lambda_{i+1}$ denotes the differential eigenvalue. If the signal contains $P$ sources, a sharp cutoff is obtained around the $P$-th eigenvalue. The eigenvalues beyond the $K$ th component have very low value as well as a low standard deviation. According to random matrix theory, the asymptotic standard deviation of the insignificant components is- $\sigma_{v}^{2}$. The signal components show high variation and the noise components have very low variation. As a result, the proposed GAP Index attains global minima at $K=P$. The number of endmembers estimated by the proposed scheme is given by-

$$
P=\arg \min _{k=1,2, ., L-3} I_{G A P}(K)
$$

As the GAP Index computation step includes the standard deviation of the eigenvalues, as a consequence perturbation of eigenvalues due to noise affects both the numerator and denominator simultaneously. We had presented the concept in a conference work (33).

Next, we utilized the GAP index-based estimator for dictionary pruning by using a two-step approach. In the first stage, we inferred the number of endmembers present in the original data by GAP index. This process identifies the number of spectral library elements to be retained. In the second stage, after we append each spectral library element with the original data and estimate the number of endmembers. The virtual
Algorithm 1 Estimation of the Number of Endmembers by Proposed GAP Index (GAP-VD)

Input: Hyperspectral image data $\mathbf{X} \in \mathbb{R}^{N \times L}$

Output: Estimated number of endmembers (virtual dimensionality) $P$

Initialization :

1: Calculate covariance matrix of the data $\mathbf{C}=$ $\frac{1}{N} \sum_{i=1}^{N}\left(x_{i}-\mu\right)^{T}\left(x_{i}-\mu\right)$ where, $\mu=\frac{1}{N} \sum_{i=1}^{N} x_{i}$ is the mean spectra

2: Find eigenvectors of and eigenvalues of the covariance matrix $\mathbf{C}$

Eigenvectors are $v=\left[v_{1}, v_{2}, \cdots v_{L}\right]$ and the corresponding eigenvalue are- $\lambda=\left[\lambda_{1}, \lambda_{2}, \cdots \lambda_{L}\right]$

3: for $i<K$ and $i \leftarrow i+1$ do

4: Calculate eigenvalue based GAP index using the formula-

$$
I_{G A P}(K)=\frac{\sigma_{\hat{\lambda}_{K+1}: \hat{\lambda}_{L-1}}}{\sigma_{\hat{\lambda}_{K}: \hat{\lambda}_{L-1}}}
$$

\section{5: end for}

6: Estimated umber of endmembers $P=$ $\arg \min _{k=1,2, ., L-3} I_{G A P}(K)$

7: return The number of endmembers $P$

dimensionality should ideally increase by one when the appended library element is not an actual image endmember and should remain unaltered if it is indeed an image endmember. We carry out the consequent pruning process using a binary hypothesis testing framework. The null hypothesis represents no change in virtual dimensionality. On the other hand, the alternative hypothesis represents the change in virtual dimensionality. Modification of virtual dimensionality indicates the absence of the specific library element, whereas, no alteration in VD signifies that the specific library element is also an image endmember.

The binary hypothesis framework is represented as-

$$
H_{0}: P_{Y_{i}}=P_{X} \text { library element is endmember }
$$

$H_{1}: P_{Y_{i}}=P_{X}+1$ library element is notendmember

\section{1) Eigen Decomposition after Rank One Modification:}

We employ rank one modification approach to compute the covariance matrix after appending each library element and speed up the process using the formulation-

$$
\hat{\mathbf{C}}_{\mathbf{i}}=\frac{1}{N} \mathbf{C}+\left(\frac{N}{N+1}\right)^{2} d_{i}{ }^{T} d_{i}
$$

\section{Abundance Computation by Low-rank and Structured Sparse Inversion}

We employ sparsity of the abundance matrix, the low-rank attribute of the abundance matrix as regularization terms in the abundance computation (27). We also utilized the structural dissimilarity between the abundance images for the inversion 


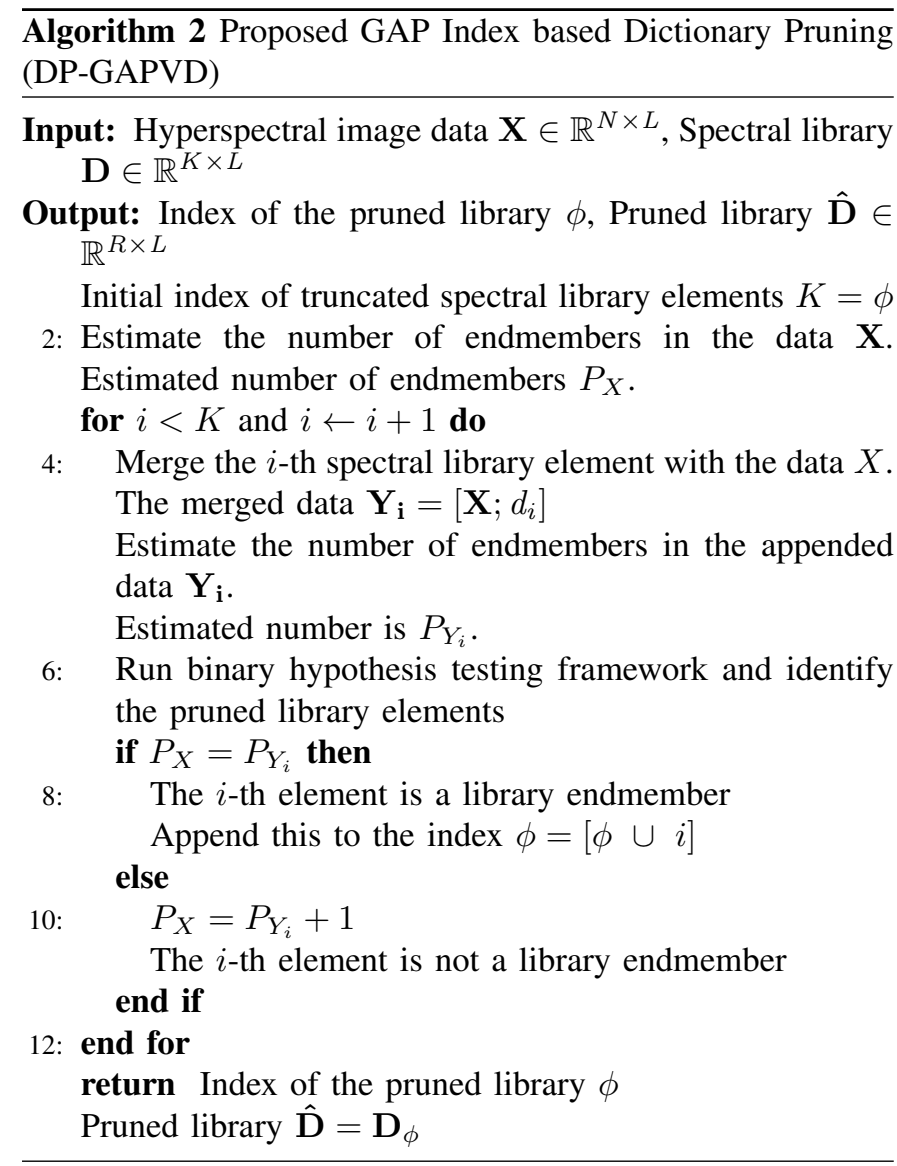

process and formulate it according to-

$$
\begin{array}{r}
\arg \min \|X-A S\|_{2}+\lambda_{1}\|A\|_{\frac{1}{2}}+\lambda_{2}\|A\|_{*} \\
+\lambda_{3} \sum_{i=1}^{P} \sum_{j=i+1}^{P} \frac{1}{F S I M\left(a_{i}, a_{j}\right)+\epsilon}
\end{array}
$$

Here, FSIM or feature similarity index (? ) denotes the structural similarity between two abundance images. The symbol $\epsilon$ used in the fourth term is a small constant employed to prevent the denominator from being divided by zero. We take $\epsilon=0.1$ in the experiments, although other values would also work.

Since, traditional $l_{2}$ norm and $l_{\frac{1}{2}}$ norm are non-differentiable and are difficult to optimize. We alleviate this issue by introducing smooth approximations of these norms. TO this aim, we approximate $l_{2}$ norm by the following function-

$$
\|X\|_{2(\mu)}=\sqrt{\left(\|X\|_{2}^{2}+\mu^{2}\right)}-\mu
$$

Here, $\mu$ is a small constraint utiulized to overcome the problem of non-differentiability at zero. We smooth $l_{\frac{1}{2}}$ norm by using the following formulation-

$$
\|X\|_{0.5}=\left(\|X\|_{0.5}-\sqrt{\mu}\right)^{2}-\mu
$$

The inclusion of low-rank property promotes sparsity in an alternative way, whereas the inclusion of image dissimilarity constraints the solution even more.

\section{EXPERIMENTAL RESULTS}

The unmixing algorithms were evaluated using several real as well as synthetic image data. In the synthetic image experiment- the number of image endmembers, noise level, and maximum abundance of any endmember in a pixel are varied.

\section{A. Performance Measures}

Performance of the unmixing algorithms are evaluated according to the following measures-

\section{- Average Spectral Angle Distance (ASAD)}

Spectral angle distance is the cosine distance between reflectance pattern of two endmemebers $s_{i}$ and $\hat{s}_{j}$ is calculated as-

$$
\operatorname{SAD}\left(s_{i}, \hat{s_{j}}\right)=\cos ^{-1}\left(\frac{\left\langle s_{i}, \hat{s_{j}}\right\rangle}{\left\|s_{i}\right\|_{2} \| \hat{s_{j} \|_{2}}}\right)
$$

The average SAD is essentially the mean SAD-

$$
A S A D(S, \hat{S})=\frac{1}{P} \sum_{i, j=1, i \neq j}^{P} \min S A D\left(s_{i}, \hat{s_{j}}\right)
$$

Lower ASAD is always wanted because, it means better match between actual and estimated endmembers.

\section{- Signal to Reconstruction Error(SRE)}

This term denotes the relative power of reconstructed data with respect to the actual data. It is given by-

$$
S R E=20 \log _{10}\left(\frac{\|\mathbf{X}\|_{2}}{\|\mathbf{X}-\hat{\mathbf{X}}\|_{2}}\right)
$$

where, $\hat{\mathbf{X}}$ is the hyperspectral data reconstructed by the unmixing or dictionary pruning algorithm. High SRE indicates better unmixing performance.

\section{- Probability of Detection(Pr Det)}

It defines the number of spectral library endmembers accurately selected. Probability of detection is defined as-

$$
\operatorname{Pr}_{D e t}(\hat{\Lambda}, \Lambda)=\operatorname{Pr}(\hat{\Lambda} \subset \Lambda)=\frac{n(\hat{\Lambda} \cap \Lambda)}{n(\Lambda)}
$$

where, $\Lambda$ represent the indices corresponding the actual spectral library elements present in the image and $\hat{\Lambda}$ is the indices corresponding to the estimated spectral library elements of the pruned library. The term $n($. identifies the cardinality of the set. The numerical value of the parameter probability of detection lies in the range $[0,1]$.Perfect match indicates $\operatorname{Pr}_{\text {Det }}=1$, and is very much desirable.

\section{B. Description of Image Data}

1) Synthetic Image Experiments:

1) Data $A 1$ : This data set is generated by Hypermix Tool (34) displayed in Figure1a. It consists of synthetic mixture of Nine spectral library elements satisfying abundance constraints. Additive white Gaussian noise is also added to the data. 


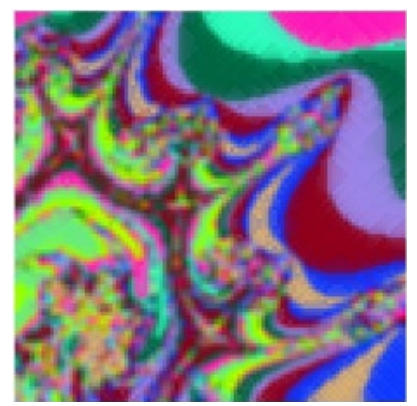

(a) RGB display with combination of bands 1,8 and 16

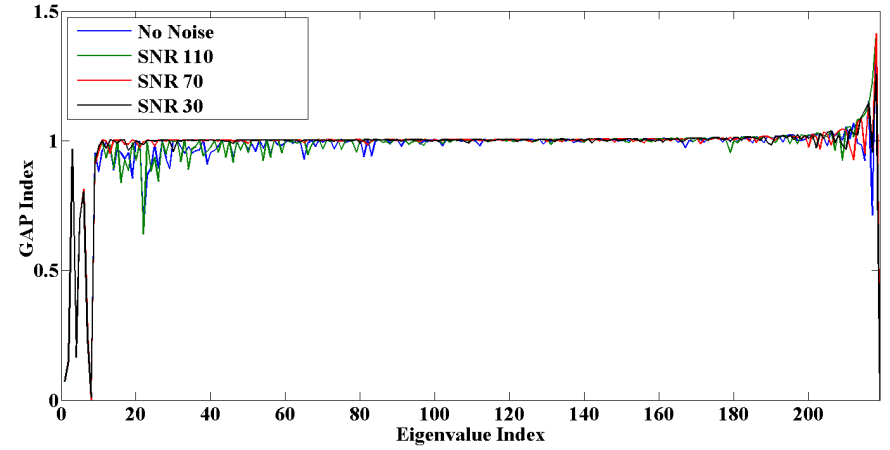

(b) Plot of GAP index

Fig. 1: Fractal1 Image

2) Data $A 2$ : This data is generated from seven library endmembers, which also include one rare endmember. The image data was generated according to convex linear mixing of the six endmembers randomly selected from USGS spectral library, which was merged with some pure pixels corresponding to the seven-th spectral library element.

3) Data $A 3$ : The abundance matrix is generated from Dirichlet distribution and endmembers are taken from USGS spectral library. The number of endmembers is varied from Five and Ten.

4) Data $A 4$ : The number of image pixels and maximum abundance of any endmember in a pixel is varied. The number of endmembers is taken at ten.

5) Data $A 5$ : In this dataset, both the number of endmembers and the noise level are varied.

6) Library $A 6$ : In this experiment, the mutual coherence of the library is varied by $1,0.8$ and 0.6 . Mutual coherence of library is varied by either removing or duplicating specific library elements.

\section{Algorithms Compared}

The virtual dimensionality estimation method is compared with state of the art algorithms such as- HFC-VD (6), HySIME (7), ELM (8), GENE-CH (35), GENE-AH (35) etc. The performance of our proposed unmixing algorithm is compared with- vertex component analysis (VCA) (36), minimum volume enclosing simplex algorithm (MVES) (37), convex hull based unmixing algorithm (TRI-P) (17), simplex identification via split augmented Lagrangian (38) and minimum volume simplex analysis (MVSA) (39). The efficiency of semi-blind unmixing is compared with- MUSIC-CSR (30), RMUSICDANSER (40), SMP (21), RSFoBa (29) and SUnSPI (25) etc.

The results shown in Table III indicate that the proposed GAP VD estimation scheme gives accurate estimation for all real images.

1) Real Image Experiments: We have compared our algorithm on three real images-Washington DC Mall image ${ }^{1}$, HYDICE Urban image ${ }^{2}$ and HYDICE Indian Pines image ${ }^{3}$.

\footnotetext{
${ }^{1}$ https://engineering.purdue.edu/ biehl/MultiSpec/hyperspectral.html

${ }^{2} \mathrm{http}: / /$ lesun.weebly.com/hyperspectral-data-set.html

${ }^{3}$ http://www.ehu.eus/ccwintco/index.php
}

- DC Mall Image DC Mall hyperspectral image (displayed in Fig.3a) contains total 210 spectral bands covering electromagnetic region $400 \mathrm{~nm}-2500 \mathrm{~nm}$. Total 188 spectral bands remain after removing the noisy and absorption bands present in the image. The USGS spectral library contains all the possible image endmembers. However, we tackle the slight mismatch in spectral resolution between the library by re-sampling (18) strategy.

- HyDICE Urban Image: This image was recorded on October 1995 from an urban area at Copperas Cove, in the U.S.A. The image has a spatial dimension of $307 \times 307$ and the spectral dimension of 210 . The image covers electromagnetic wavelengths $400 \mathrm{~nm}$ to $2500 \mathrm{~nm}$. According to the ground truth study (41) the image contains four dominant endmembers- Asphalt Road, Grass, Tree and Roof. However, there is a slight mismatch in spectral resolution between the reflectance profile of the spectral library elements and the image pixels.

\section{Results: Estimation of Number of Endmembers}

In Fig.1b we plotted the proposed GAP index for Fractall image generated by data $A 1$. The plot indicates that the proposed GAP index attains global minima for nine endmembers, which is indeed the actual number of endmembers. Presence of noise has a negligible effect on this parameter.

We displayed the comparative study of virtual dimensionality of Fractal1 image data $A 1$ in Table I. Only GAP-VD yields an accurate estimation of different observation. The effectiveness of algorithms on the presence of rare endmembers is displayed in Table II. This experiment uses the data generated by $A 2$. The results exhibit that only GAP-VD leads to accurate estimation, even when the data is highly mixed. Among the other algorithms, HYSIME (7) is the closest. We tabulated the results of real images experiment in Table III, which suggests that only GAP-VD can estimate the number of endmembers accurately.

The runtime of VD estimation of algorithms are evaluated in an Intel $i 5$ processor with 16GB RAM on Matlab 2016(a) platform. The average runtime of a total of 10 iterations is considered for comparison. We can conclude from Fig. 2a. that HFC-VD (6) is the fastest scheme closely followed by GAP-VD, other estimators have a significantly higher 
TABLE I: Estimation of the Number of Endmembers for Data $A 1$. Actual Number is Nine.

\begin{tabular}{|c|c|c|c|c|c|c|}
\hline SNR (in dB) & HFC-VD & HySIME & ELM & GENE-CH & GENE-AH & GAP-VD \\
\hline No Noise & 8 & $\mathbf{9}$ & $\mathbf{9}$ & 6 & 6 & $\mathbf{9}$ \\
\hline 70 & 8 & $\mathbf{9}$ & $\mathbf{9}$ & 5 & 6 & $\mathbf{9}$ \\
\hline 50 & 8 & $\mathbf{9}$ & $\mathbf{9}$ & 5 & 6 & $\mathbf{9}$ \\
\hline 30 & 8 & 8 & 13 & 5 & 5 & $\mathbf{9}$ \\
\hline 10 & 10 & 7 & 15 & 5 & 4 & $\mathbf{9}$ \\
\hline
\end{tabular}

TABLE II: Estimation of the Number of Endmembers for Data $A 2$. The total number of endmembers including the rare endmembers is seven

\begin{tabular}{|c|c|c|c|c|c|c|c|}
\hline${ }_{\text {nPixels }}$ & Max Abun & HFC-VD & HySIME & ELM & GENE-CH & GENE-AH & GAP-VD \\
\hline \multirow{4}{*}{1000} & 1 & 5 & 7 & 7 & 3 & 2 & 7 \\
\hline & 0.8 & 5 & 7 & 6 & 3 & 4 & 7 \\
\hline & 0.6 & 6 & 7 & 7 & 3 & 3 & 7 \\
\hline & 0.4 & 7 & 8 & 7 & 4 & 3 & 7 \\
\hline \multirow{4}{*}{500} & 1 & 2 & 7 & 7 & 5 & 4 & 7 \\
\hline & 0.8 & 2 & 7 & 7 & 5 & 5 & 7 \\
\hline & 0.6 & 4 & 6 & 7 & 4 & 5 & 7 \\
\hline & 0.4 & 5 & 7 & 8 & 4 & 4 & 7 \\
\hline \multirow{4}{*}{200} & 1 & 4 & 7 & 7 & 5 & 6 & 7 \\
\hline & 0.8 & 2 & 7 & 7 & 5 & 5 & 7 \\
\hline & 0.6 & 3 & 7 & 7 & 3 & 5 & 7 \\
\hline & 0.4 & 3 & 7 & 6 & 3 & 4 & 7 \\
\hline \multirow{4}{*}{100} & 1 & 2 & 6 & 7 & 3 & 3 & 7 \\
\hline & 0.8 & 4 & 7 & 6 & 3 & 2 & 7 \\
\hline & 0.6 & 2 & 7 & 6 & 2 & 2 & 7 \\
\hline & 0.4 & 2 & 7 & 7 & 3 & 2 & 7 \\
\hline
\end{tabular}

TABLE III: Estimation of Number of Endmembers for Real Images

\begin{tabular}{|c|c|c|c|c|c|c|c|}
\hline Image & HFC-VD & HySIME & ELM & GENE-CH & GENE-AH & GAP-VD & Actual \\
\hline DC Mall & 8 & 7 & 6 & 5 & 4 & $\mathbf{5}$ & $\mathbf{5}$ \\
\hline Indian Pines & 21 & 14 & 16 & 5 & 8 & $\mathbf{1 3}$ & $\mathbf{1 2}$ \\
\hline Urban & 25 & 9 & 8 & 3 & 2 & $\mathbf{4}$ & $\mathbf{4}$ \\
\hline & \multicolumn{7}{|l}{}
\end{tabular}

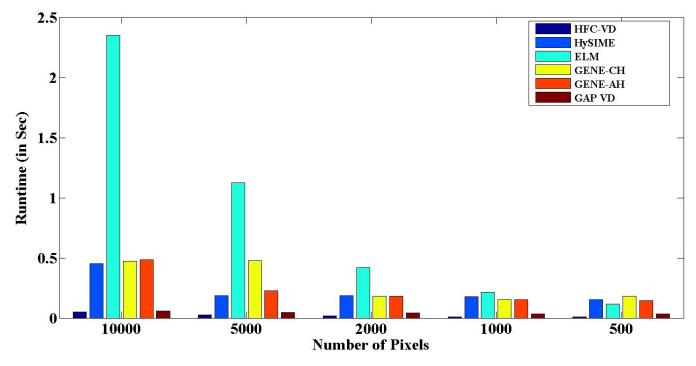

(a) Runtime comparison of synthetic data $A 3$

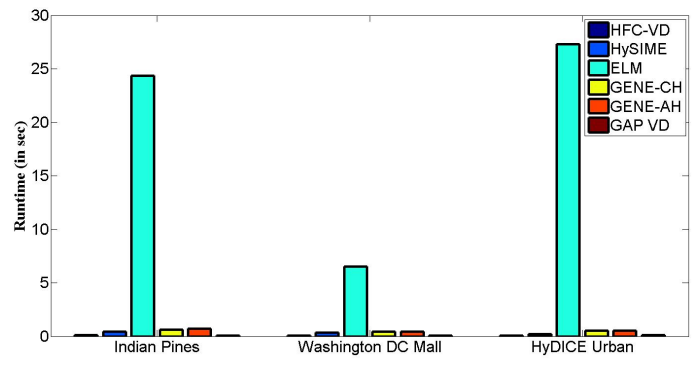

(b) Runtime comparison of real images

Fig. 2: Comparing runtime of virtual dimensionality estimation methods

computational runtime. Comparison of average runtime shown in Fig.2b stipulates that GAP-VD and HFC-VD method (6) have significantly lower runtime compared to other estimation schemes. Though HFC-VD (6) is the fastest. GAP-VD offers other advantages. The runtime comparison on real data shown in Figure. $2 b$ also points out that HFC-VD (6) is the fastest followed by GAP-VD.

\section{E. Results: Semi-blind Unmixing}

The comparison of average SAD (ASAD) and SRE on data $A 4$ is shown in Table IV and Table V respectively. It highlights that GAP-VD corresponds to lower ASAD and higher SRE. Comparison of the probability of detection shown in Table. VI illustrates that our proposed GAP-VD has a noticeably higher probability of detection. In most cases, the value is close to 
Fig. 3: RGB display of real images

TABLE IV: Comparison of Average Spectral Angle Distance (ASAD) between Actual and Estimated Endmembers on Data $A 4$

\begin{tabular}{|c|c|c|c|c|c|c|c|}
\hline nPixel & Max Abun & VCA & NABO & TRIP & SISAL & MVSA & DP-GAPVD \\
\hline \multirow{5}{*}{1000} & 1 & 0.0649 & 0.0640 & 0.0724 & 0.0165 & 0.0877 & $\mathbf{5 . 2 6 7 e - 0 4}$ \\
& 0.8 & 0.0071 & 0.0732 & 0.0794 & 0.0119 & 0.0467 & $\mathbf{7 . 9 4 3 e - 0 4}$ \\
& 0.6 & 0.0909 & 0.0847 & 0.0882 & 0.0125 & 0.0177 & $\mathbf{5 . 9 2 3 2 e - 0 4}$ \\
& 0.4 & 0.1063 & 0.1057 & 0.1033 & 0.0111 & 0.2419 & $\mathbf{5 . 6 0 8 e - 0 4}$ \\
\hline \multirow{5}{*}{500} & 1 & 0.0872 & 0.0776 & 0.0823 & 0.0262 & 0.0645 & $\mathbf{4 . 9 7 8 e - 0 4}$ \\
& 0.8 & 0.1095 & 0.0979 & 0.1161 & .0259 & 0.3668 & $\mathbf{4 . 9 2 3 e - 0 4}$ \\
& 0.6 & 0.132 & 0.1181 & 0.123 & 0.0227 & 0.1225 & $\mathbf{6 . 1 9 1 e - 0 3}$ \\
& 0.4 & 0.1302 & 0.1226 & 0.1226 & 0.0911 & 0.1302 & $\mathbf{5 . 7 7 9 e - 0 3}$ \\
\hline \multirow{5}{*}{200} & 1 & .0753 & 0.0776 & 0.0883 & 0.1297 & 0.1274 & $\mathbf{4 . 6 2 3 e - 0 3}$ \\
& 0.8 & 0.1139 & 0.1003 & 0.0962 & 0.1723 & 0.1206 & $\mathbf{4 . 5 3 8 e - 0 3}$ \\
& 0.6 & 0.0628 & 0.0574 & 0.0560 & 0.1725 & 0.1681 & $\mathbf{4 . 2 6 2 e - 0 3}$ \\
& 0.4 & 0.0935 & 0.0991 & 0.0852 & 0.0936 & 0.2290 & $\mathbf{4 . 0 3 2 e - 0 3}$ \\
\hline
\end{tabular}

TABLE V: Comparison of Signal to Reconstruction Error (SRE) for Data $A 4$

\begin{tabular}{|c|c|c|c|c|c|c|c|}
\hline nPixel & Max Abun & MUSIC-CSR & RMUSIC & SMP & RSFoBa & SUnSPI & DP-GAPVD \\
\hline \multirow{3}{*}{1000} & 1 & 89.703 & 38.787 & 29.506 & 30.906 & 32.569 & $\mathbf{1 1 3 . 6 9 5 . 0 1 6}$ \\
& 0.8 & 94.828 & 39.184 & 35.2071 & 31.281 & 31.545 & $\mathbf{1 1 0 . 0 7 7}$ \\
& 0.6 & 97.774 & 32.509 & 30.569 & 30.776 & 31.148 & $\mathbf{1 0 6 . 3 1 8}$ \\
\hline \multirow{3}{*}{500} & 1 & 98.038 & 35.061 & 31.994 & 32.028 & 33.164 & $\mathbf{1 2 0 . 6 8 4}$ \\
& 0.8 & 102.531 & 31.023 & 27.769 & 31.369 & 31.427 & $\mathbf{1 1 5 . 5 2 1}$ \\
& 0.6 & 119.217 & 34.595 & 30.283 & 32.214 & 30.955 & $\mathbf{1 0 8 . 1 0 3}$ \\
\hline
\end{tabular}

TABLE VI: Comparison of Probability of Detection for Data $A 4$

\begin{tabular}{|c|c|c|c|c|c|c|c|}
\hline nPixel & Max Abun & MUSIC-CSR & RMUSIC & SMP & RSFoBa & SUnSPI & DP-GAPVD \\
\hline \multirow{3}{*}{1000} & 1 & $\mathbf{1}$ & 0.6 & 0.8 & 0.1724 & 0.2083 & $\mathbf{1}$ \\
& 0.8 & 0.9 & $\mathbf{1}$ & 0.8 & 0.1724 & 0.2 & $\mathbf{1}$ \\
& 0.6 & $\mathbf{1}$ & 0.6 & 0.8 & 0.1613 & 0.1852 & $\mathbf{1}$ \\
\hline \multirow{3}{*}{500} & 1 & $\mathbf{1}$ & $\mathbf{1}$ & 0.8 & 0.1563 & 0.2381 & $\mathbf{1}$ \\
& 0.8 & $\mathbf{1}$ & 0.8 & 0.8 & 0.1926 & 0.2083 & $\mathbf{1}$ \\
& 0.6 & 0.9 & 0.8 & 0.8 & 0.1852 & 0.1926 & $\mathbf{1}$ \\
\hline
\end{tabular}

TABLE VII: Comparison of Signal to Reconstruction Error (SRE) for Synthetic Data $A 5$

\begin{tabular}{|c|c|c|c|c|c|c|c|}
\hline nEM & Noise (SNR in dB) & MUSIC-CSR & RMUSIC & SMP & RSFoBa & SUnSPI & DP-GAPVD \\
\hline \multirow{4}{*}{5} & No Noise & 100.897 & 41.286 & 36.131 & 30.118 & 32.191 & $\mathbf{1 1 1 . 3 0 8}$ \\
& 70 & 63.243 & 37.928 & 36.111 & 31.708 & 30.916 & $\mathbf{7 3 . 3 2 6}$ \\
& 50 & 43.692 & 34.369 & 38.549 & 31.787 & 30.717 & $\mathbf{4 7 . 0 1 5}$ \\
& 30 & 35.692 & 35.083 & 30.292 & 31.094 & 31.107 & $\mathbf{4 4 . 6 5 7}$ \\
\hline \multirow{4}{*}{10} & No Noise & 104.928 & 35.441 & 35.869 & 30.681 & 31.208 & $\mathbf{1 1 0 . 9 3 4}$ \\
& 70 & 42.201 & 32.769 & 34.567 & 30.824 & 31.115 & $\mathbf{6 7 . 2 6 7}$ \\
& 50 & 35.378 & 32.387 & 32.711 & 30.164 & 29.681 & $\mathbf{3 9 . 3 0 8}$ \\
\hline
\end{tabular}

1 , which indicates exact dictionary pruning. SRE and Pr Det values obtained on data $A 5$ are displayed in Table VII and VIII respectively. Our proposed DP GAP-VD algorithm performs significantly better than prevalent unmixing algorithms, even if a significant amount of noise is present in the data. Initial noise removal will further improve the unmixing performance. The proposed GAP-VD based estimation scheme results into unity probability of detection in most of the situations. This result ascertains the superior performance of the proposed workflow. Table. IX and Table. $X$ exhibit unmixing performance in the presence of a spectral library with high mutual coherence. Our proposed GAP-VD achieves almost unity probability of 
TABLE VIII: Comparison of Probability of Detection for Data $A 5$

\begin{tabular}{|c|c|c|c|c|c|c|c|}
\hline $\mathrm{nEm}$ & SNR (in dB) & MUSIC-CSR & RMUSIC & SMP & RSFoBa & SUnSPI & GAP-VD \\
\hline \multirow{5}{*}{5} & No Noise & $\mathbf{1}$ & 0.6 & 0.8 & 0.1923 & 0.1724 & $\mathbf{1}$ \\
& 70 & $\mathbf{1}$ & 0.4 & 0.8 & 0.1613 & 0.1563 & $\mathbf{1}$ \\
& 50 & 0.9 & 0.4 & 0.6 & 0.1515 & 0.1563 & $\mathbf{1}$ \\
& 30 & 0.9 & 0.2 & 0.6 & 0.1471 & 0.1429 & $\mathbf{1}$ \\
\hline \multirow{5}{*}{10} & No Noise & $\mathbf{1}$ & 0.2 & 0.5 & 0.303 & 0.2381 & $\mathbf{1}$ \\
& 70 & $\mathbf{1}$ & 0.4 & 0.5 & 0.2703 & 0.222 & $\mathbf{1}$ \\
& 50 & $\mathbf{0 . 9}$ & 0.2 & 0.5 & 0.2632 & 0.1961 & $\mathbf{0 . 9}$ \\
& 30 & 0.8 & 0.4 & 0.5 & 0.2326 & 0.1887 & $\mathbf{1}$ \\
\hline
\end{tabular}

TABLE IX: Comparison of probability of detection (Pr Det) for data $A 6$

\begin{tabular}{|c|c|c|c|c|c|c|}
\hline Mutual Coherence & MUSIC-CSR & RMUSIC & SMP & RSFoBa & SUnSPI & GAP-VD \\
\hline 1 & 0.8 & 0.6 & 0.8 & 0.1724 & 0.2083 & $\mathbf{0 . 9}$ \\
0.8 & 0.9 & 0.2 & 0.8 & 0.1724 & 0.2 & $\mathbf{1}$ \\
0.6 & 0.8 & 0.3 & 0.6 & 0.1613 & 0.1852 & $\mathbf{1}$ \\
\hline
\end{tabular}

TABLE X: Comparison of SRE for Synthetic Data $A 6$

\begin{tabular}{|c|c|c|c|c|c|c|}
\hline Mutual Coherence & MUSIC-CSR & RMUSIC & SMP & RSFoBa & SUnSPI & GAP-VD \\
\hline 1 & 77.293 & 35.416 & 37.152 & 38.681 & 32.253 & $\mathbf{8 8 . 1 7 2}$ \\
0.8 & 79.453 & 37.218 & 42.257 & 39.694 & 42.521 & $\mathbf{8 5 . 5 0 2}$ \\
0.6 & 79.694 & 47.042 & 44.166 & 39.581 & 42.331 & $\mathbf{8 4 . 6 9 8}$ \\
\hline
\end{tabular}

TABLE XI: Comparison of Unmixing Performance in Real Images

\begin{tabular}{|c|c|c|c|c|c|c|}
\hline DC Mall Image & MUSIC-CSR & RMUSIC & SMP & RSFoBa & SUnSPI & GAP-VD \\
\hline Pr Det & 0.617 & 0.2941 & 0.228 & 0.1852 & 0.2083 & 1 \\
SRE & 81.208 & 37.569 & 39.967 & 33.027 & 34.107 & $\mathbf{1 1 8 . 6 9 2}$ \\
\hline HyDICE Urban Image & & & & & & \\
\hline Pr Det & 0.667 & 0.3636 & 0.3077 & 0.2105 & 0.1905 & 1 \\
SRE & 81.549 & 39.404 & 37.098 & 35.367 & 37.458 & $\mathbf{1 1 2 . 3 7 6}$ \\
\hline
\end{tabular}

detection and comparatively higher SRE in this scenario. The results on real image experiment displayed in Table XI, illustrates that our proposed DP GAP-VD based unmixing results in unity probability of detection as well as higher SRE compared to other methods. We can attribute the improved performance to two main factors- the estimator estimates the number of endmembers accurately even in the presence of noise, and the proposed sparse inversion method exploits the main attributes of the data namely low-rankness and structural similarity.

\section{Conclusion ANd Future Scope}

This paper proposes a non-parametric scheme for performing semi-blind unmixing using library pruning strategy. The virtual dimensionality estimation is seminal to the proposed dictionary pruning concept. Since, library pruning methods which incorporate an estimation of the number of endmembers identify the exact set of endmembers, these methods are extremely accurate. This is a major improvement from most of the prevalent pruning schemes as these methods often identify a pruned library with high cardinality. We propose a improved noise robust index for estimating VD, which makes the whole unmixing itself robust to noise. Our proposed VD estimation method is capable of producing accurate estimation in presence of rare endmembers and lower number of pixels. Simulated and real image experiments demonstrate the efficacy and noise robustness of our proposed method in diverse scenarios, with affordable computational complexity.These results also establish the superiority of our proposed estimator in identifying rare endmembers.

Our proposed workflow is pertinent for linear mixing, because, estimation of virtual dimensionality for nonlinear mixing is a trivial task in itself. In turn, the virtual dimensionality approach can be applied when the data is converted to piecewise linear segments. In future, we can further explore the approaches to accurately estimate the number of endmembers for the nonlinear mixture of endmembers.

\section{REFERENCES}

[1] D. Landgrebe, Hyperspectral image data analysis. IEEE, 2002, vol. 19, no. 1.

[2] S. Das, S. Bhattacharya, and P. K. Khatri, "Feature extraction approach for quality assessment of remotely sensed hyperspectral images," Journal of Applied Remote Sensing, vol. 14, no. 2, p. 026514, 2020.

[3] W.-K. Ma, J. M. Bioucas-Dias, T.-H. Chan, N. Gillis, P. Gader, A. J. Plaza, A. Ambikapathi, and C.-Y. Chi, "A signal processing perspective on hyperspectral unmixing: 
Insights from remote sensing," IEEE Signal Processing Magazine, vol. 31, no. 1, pp. 67-81, 2014.

[4] S. Das, A. Routray, and A. K. Deb, "Efficient tensor decomposition approach for estimation of the number of endmembers in a hyperspectral image," Journal of Applied Remote Sensing, vol. 14, no. 1, p. 016519, 2020.

[5] S. Das, S. Chakraborty, A. Routray, and A. K. Deb, "Library-aided bilinear unmixing of hyperspectral image using subspace clustering and multistep pruning," Journal of Applied Remote Sensing, vol. 13, no. 4, p. 046506, 2019.

[6] C.-I. Chang and Q. Du, "Estimation of number of spectrally distinct signal sources in hyperspectral imagery," IEEE Transactions on geoscience and remote sensing, vol. 42, no. 3, pp. 608-619, 2004.

[7] J. M. Bioucas-Dias and J. M. Nascimento, "Hyperspectral subspace identification," IEEE Transactions on Geoscience and Remote Sensing, vol. 46, no. 8, pp. 2435-2445, 2008.

[8] B. Luo, J. Chanussot, S. Douté, and L. Zhang, "Empirical automatic estimation of the number of endmembers in hyperspectral images," IEEE Geoscience and Remote Sensing Letters, vol. 10, no. 1, pp. 24-28, 2013.

[9] S. Das, J. N. Kundu, and A. Routray, "Estimation of number of endmembers in a hyperspectral image using eigen thresholding," in India Conference (INDICON), 2015 Annual IEEE. IEEE, 2015, pp. 1-5.

[10] H. Asadi and B. Seyfe, "Source number estimation via entropy estimation of eigenvalues (eee) in gaussian and non-gaussian noise," arXiv preprint arXiv:1311.6051, 2013.

[11] A. Sumarsono and Q. Du, "Low-rank subspace representation for estimating the number of signal subspaces in hyperspectral imagery," IEEE Transactions on Geoscience and Remote Sensing, vol. 53, no. 11, pp. 62866292, 2015.

[12] H. Akaike, "A new look at the statistical model identification," IEEE transactions on automatic control, vol. 19, no. 6, pp. 716-723, 1974.

[13] M. H. Hansen and B. Yu, "Model selection and the principle of minimum description length," Journal of the American Statistical Association, vol. 96, no. 454, pp. 746-774, 2001.

[14] C.-I. Chang, W. Xiong, H.-M. Chen, and J.-W. Chai, "Maximum orthogonal subspace projection approach to estimating the number of spectral signal sources in hyperspectral imagery," IEEE Journal of Selected Topics in Signal Processing, vol. 5, no. 3, pp. 504-520, 2011.

[15] C.-I. Chang, W. Xiong, and C.-H. Wen, "A theory of high-order statistics-based virtual dimensionality for hyperspectral imagery," IEEE Transactions on Geoscience and Remote Sensing, vol. 52, no. 1, pp. 188-208, 2014.

[16] B. Rasti, M. O. Ulfarsson, and J. R. Sveinsson, "Hyperspectral subspace identification using sure," IEEE Geoscience and Remote Sensing Letters, vol. 12, no. 12, pp. 2481-2485, 2015.

[17] A. Ambikapathi, T.-H. Chan, and C.-Y. Chi, "Convex geometry based estimation of number of endmembers in hyperspectral images," in Acoustics, Speech and Signal Processing (ICASSP), 2012 IEEE International Conference on. IEEE, 2012, pp. 1233-1236.

[18] M.-D. Iordache, J. M. Bioucas-Dias, and A. Plaza, "Sparse unmixing of hyperspectral data," IEEE Transactions on Geoscience and Remote Sensing, vol. 49, no. 6, pp. 2014-2039, 2011.

[19] N. Akhtar, F. Shafait, and A. Mian, "Futuristic greedy approach to sparse unmixing of hyperspectral data," IEEE Transactions on Geoscience and Remote Sensing, vol. 53, no. 4, pp. 2157-2174, 2015.

[20] S. Das and A. Routray, "Covariance similarity approach for semiblind unmixing of hyperspectral image," IEEE Geoscience and Remote Sensing Letters, vol. 16, no. 6, pp. 937-941, 2019.

[21] Z. Shi, W. Tang, Z. Duren, and Z. Jiang, "Subspace matching pursuit for sparse unmixing of hyperspectral data," IEEE Transactions on Geoscience and Remote Sensing, vol. 52, no. 6, pp. 3256-3274, 2014.

[22] W. Dai and O. Milenkovic, "Subspace pursuit for compressive sensing signal reconstruction," IEEE Transactions on Information Theory, vol. 55, no. 5, pp. 2230 2249, 2009.

[23] S. Das, A. Routray, and A. K. Deb, "Sparsity measure based library aided unmixing of hyperspectral image," IET Image Processing, vol. 13, no. 8, pp. 1364-1372, 2019.

[24] J. A. Tropp, A. C. Gilbert, and M. J. Strauss, "Simultaneous sparse approximation via greedy pursuit," in Acoustics, Speech, and Signal Processing, 2005. Proceedings.(ICASSP'05). IEEE International Conference on, vol. 5. IEEE, 2005, pp. v-721.

[25] W. Tang, Z. Shi, Y. Wu, and C. Zhang, "Sparse unmixing of hyperspectral data using spectral a priori information," IEEE Transactions on Geoscience and Remote Sensing, vol. 53, no. 2, pp. 770-783, 2015.

[26] R. Wang, H.-C. Li, W. Liao, X. Huang, and W. Philips, "Centralized collaborative sparse unmixing for hyperspectral images," IEEE Journal of Selected Topics in Applied Earth Observations and Remote Sensing, vol. 10, no. 5, pp. 1949-1962, 2017.

[27] S. Das, S. Chakraborty, A. Routray, and A. K. Deb, "Fast linear unmixing of hyperspectral image by slow feature analysis and simplex volume ratio approach," in IGARSS 2019-2019 IEEE International Geoscience and Remote Sensing Symposium. IEEE, 2019, pp. 560-563.

[28] X.-L. Zhao, F. Wang, T.-Z. Huang, M. K. Ng, and R. J. Plemmons, "Deblurring and sparse unmixing for hyperspectral images," IEEE Transactions on Geoscience and Remote Sensing, vol. 51, no. 7, pp. 4045-4058, 2013.

[29] W. Tang, Z. Shi, and Y. Wu, "Regularized simultaneous forward-backward greedy algorithm for sparse unmixing of hyperspectral data," IEEE Transactions on Geoscience and Remote Sensing, vol. 52, no. 9, pp. 5271-5288, 2014.

[30] M.-D. Iordache, J. M. Bioucas-Dias, A. Plaza, and B. Somers, "Music-csr: Hyperspectral unmixing via multiple signal classification and collaborative sparse regression," IEEE Transactions on Geoscience and Remote 
Sensing, vol. 52, no. 7, pp. 4364-4382, 2014.

[31] N. Acito, M. Diani, and G. Corsini, "Signal-dependent noise modeling and model parameter estimation in hyperspectral images," IEEE Transactions on Geoscience and Remote Sensing, vol. 49, no. 8, pp. 2957-2971, 2011.

[32] B. Nadler, F. Penna, and R. Garello, "Performance of eigenvalue-based signal detectors with known and unknown noise level," in Communications (ICC), 2011 IEEE International Conference on. IEEE, 2011, pp. $1-5$.

[33] S. Das, A. Routray, and A. K. Deb, "Noise robust estimation of number of endmembers in a hyperspectral image by eigenvalue based gap index," in Hyperspectral Image and Signal Processing: Evolution in Remote Sensing (WHISPERS), 2016 8th Workshop on. IEEE, 2016, pp. $1-5$.

[34] L.-I. Jiménez, G. Martin, and A. Plaza, "Hypermix: A new tool for quantitative evaluation of end member identification and spectral unmixing techniques," in Geoscience and Remote Sensing Symposium (IGARSS), 2012 IEEE International. IEEE, 2012, pp. 1393-1396.

[35] A. Ambikapathi, T.-H. Chan, C.-Y. Chi, and K. Keizer, "Hyperspectral data geometry-based estimation of number of endmembers using p-norm-based pure pixel identification algorithm," IEEE Transactions on Geoscience and Remote Sensing, vol. 51, no. 5, pp. 2753-2769, 2013.

[36] J. M. Nascimento and J. M. Dias, "Vertex component analysis: A fast algorithm to unmix hyperspectral data," IEEE transactions on Geoscience and Remote Sensing, vol. 43, no. 4, pp. 898-910, 2005.

[37] T.-H. Chan, C.-Y. Chi, Y.-M. Huang, and W.-K. Ma, "A convex analysis-based minimum-volume enclosing simplex algorithm for hyperspectral unmixing," IEEE Transactions on Signal Processing, vol. 57, no. 11, pp. 4418-4432, 2009.

[38] J. M. Bioucas-Dias, "A variable splitting augmented lagrangian approach to linear spectral unmixing," in Hyperspectral Image and Signal Processing: Evolution in Remote Sensing, 2009. WHISPERS'09. First Workshop on. IEEE, 2009, pp. 1-4.

[39] J. Li and J. M. Bioucas-Dias, "Minimum volume simplex analysis: A fast algorithm to unmix hyperspectral data," in Geoscience and Remote Sensing Symposium, 2008. IGARSS 2008. IEEE International, vol. 3. IEEE, 2008, pp. III-250.

[40] X. Fu, W.-K. Ma, J. M. Bioucas-Dias, and T.-H. Chan, "Semiblind hyperspectral unmixing in the presence of spectral library mismatches," IEEE Transactions on Geoscience and Remote Sensing, vol. 54, no. 9, pp. 51715184, 2016.

[41] F. Zhu, "Hyperspectral unmixing: Ground truth labeling, datasets, benchmark performances and survey." 